\title{
RF Performance of T-DAB Receivers
}

\author{
Roel Schiphorst, Jordy Potman, Klaas L. Hofstra, Harm S. Cronie, and Cornelis H. Slump
}

\begin{abstract}
In every wireless system, the weakest link determines the performance of the network. In this paper the Radio Frequency (RF) performance of both band III and L-band Terrestrial Digital Audio Broadcasting (T-DAB) consumer receivers are discussed. The receivers have been tested based on the EN 50248 standard. The test results show that the average consumer receiver for band III meets the requirements set by EN 50248, except for the non-adjacent interferer experiments. In this experiment, the average consumer receiver performs up to $10 \mathrm{~dB}$ worse than required. In addition, the experiments reveal that there is a large difference in performance between consumer receivers. Besides band III, also L-band consumer receivers have been evaluated. The results of the L-band experiments show that the consumer receivers are not capable of decoding a DAB signal with a COST207 rural area channel model in case of T-DAB mode IV. Network operators should for this reason use mode II for the L-band and should expect a larger influence of non-adjacent interference on receiver performance in band III than anticipated based on EN 50248.
\end{abstract}

Index Terms-Adjacent channel interference, band III, consumer receiver, EN 50248, in-band interference, L-band, nonadjacent channel interference, sensitivity, T-DAB.

\section{INTRODUCTION}

$\mathbf{T}$ HE COVERAGE of a broadcasting network is determined both by the network design and the Radio Frequency (RF) performance (such as sensitivity, selectivity) of the receivers. In this paper the RF performance of Terrestrial Digital Audio Broadcasting (T-DAB) receivers for both band $\mathrm{III}^{1}$ and the L-band ${ }^{2}$ are discussed. There is not much previous work on the performance of T-DAB consumer receivers. In Canada [1] the performance of L-band receivers was investigated for an audio sub channel of $224 \mathrm{kbps}$ using protection level UEP3 ${ }^{3}$ [2].

In our receiver experiments, we tested 15 consumer receivers, which include different models (personal, portable and $\mathrm{Hi}-\mathrm{Fi}$ ) and chipsets ${ }^{4}$. Seven receivers are also able to receive the L-band. The evaluated receivers covered about 80 percent

\footnotetext{
Manuscript received August 6, 2007; revised December 21, 2007. This work was supported by the Dutch Ministry of Economic Affairs.

The authors are with the University of Twente, Enschede, the Netherlands (e-mail r.schiphorst@ewi.utwente.nl; j.potman@ewi.utwente.nl; k.1.hofstra@ewi.utwente.nl; h.s.cronie@ewi.utwente.nl; c.h.slump@ewi. utwente.nl).

Color versions of one or more of the figures in this paper are available online at http://ieeexplore.iee.org.

Digital Object Identifier 10.1109/TBC.2008.917275

${ }^{1} \mathrm{Band} \mathrm{III}$ is the frequency region between 170 and $240 \mathrm{MHz}$

${ }^{2}$ The L-band is the frequency region between 1452 and $1492 \mathrm{MHz}$.

${ }^{3}$ In DAB two different kinds of error protection are defined: Unequal Error Protection (UEP) used for audio sub channels and Equal Error Protection (EEP) tailored for data services. In each mode, several protection levels are defined. A protection level is an indication for the used code rate.

${ }^{4} \mathrm{~A}$ chipset is a set of electronic components responsible for decoding the T-DAB signal into an audio signal.
}

of the market in the Netherlands in 2005. In other countries, more DAB receiver models were available. However, the RF performance of a T-DAB receiver is mainly determined by the DAB chipset. And the majority of the market contains only a few DAB chipsets which were included in our experiments. For that reason, it is expected that testing more receivers which were available in 2005 , would not have a large influence on our results.

The DAB chipset contains both an analog front-end and a baseband chip for digital signal processing. Both the design of the front-end and the used digital signal processing algorithms determine the RF performance of a receiver. Information about trade-offs in a DAB receiver can be found in [3].

This paper only discusses the performance of the median receiver (both band III and L-band), which is compared to the EN 50248 requirements [4]. The median receiver is defined as the receiver where $50 \%$ of the tested receivers have a better performance and $50 \%$ a worse performance.

The receivers are evaluated in four different RF tests [4]:

1) A test to determine the sensitivity of a receiver i.e. sensitivity experiment.

2) A test to evaluate how the receiver can cope with strong adjacent channel interference, i.e. adjacent interference experiment.

3) A test to evaluate how the receiver can cope with strong non-adjacent channel interference, i.e. non-adjacent interference experiment.

4) A test to evaluate how the receiver can cope with in-band interference i.e. in-band interference experiment.

In addition, we measured the antenna factor of consumer band III antennas.

\section{A. Contributions}

The main contributions of this paper are:

- Evaluating the RF performance of the median band III T-DAB consumer receiver by experimental results. These experiments are based on EN 50248.

- Evaluating the RF performance of the median L-band T-DAB consumer receiver by experimental results.

- Measuring the antenna factor of consumer band III receivers.

- Deriving the typical sensitivity for both a band III and L-band T-DAB receiver.

In the next section, the test setup for testing the consumer receivers is discussed. In the remaining sections, the consumer receiver experiments are described and differences with the experiments in EN 50248 are indicated. The paper ends with the conclusions. 


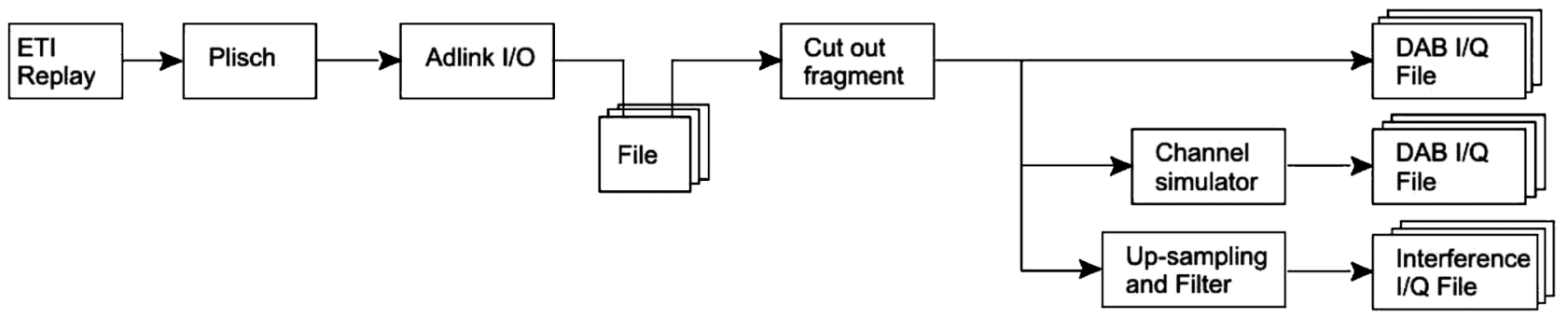

Fig. 1. Block scheme for DAB signal capture.

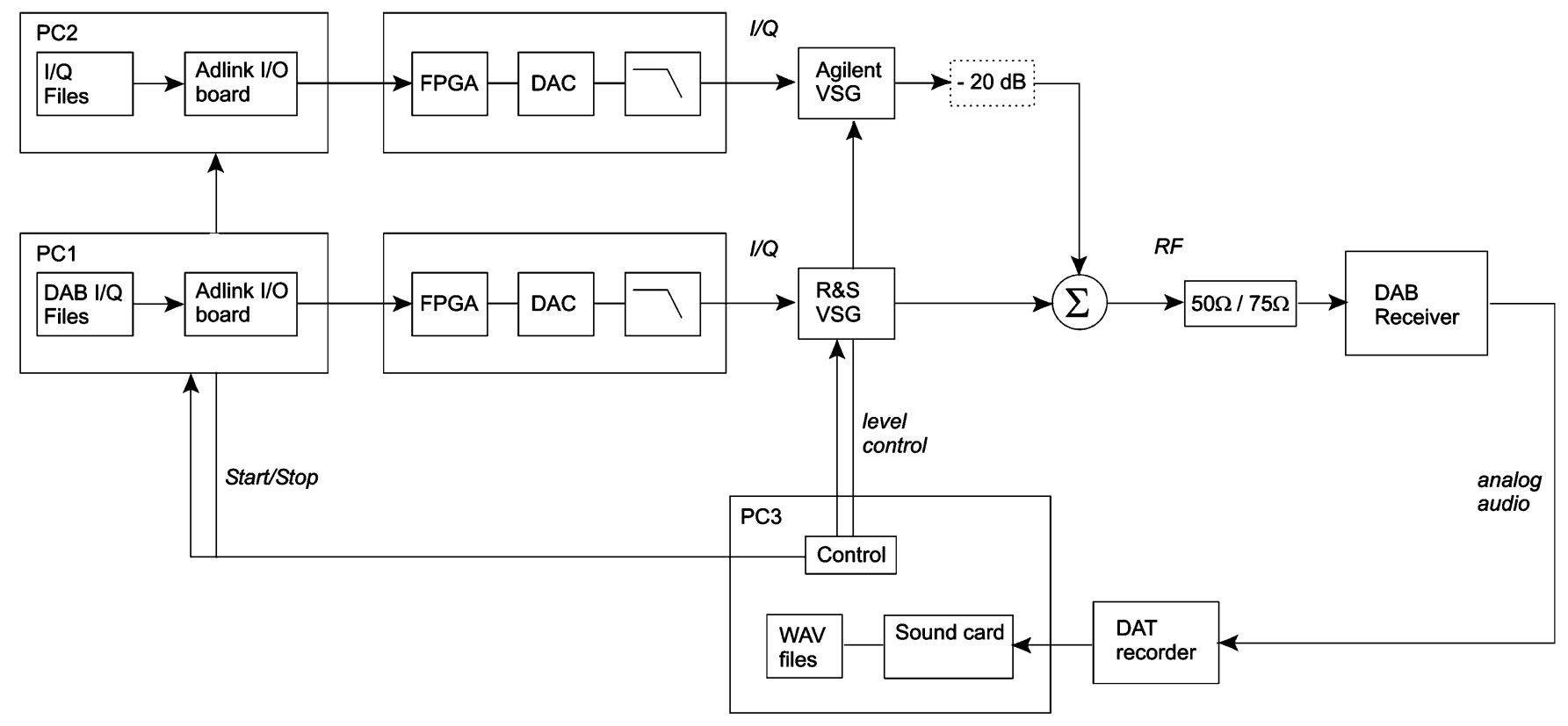

Fig. 2. Block scheme of the test setup for RF experiments.

\section{MeAsurement Setup FOR Testing T-DAB CONSUMER RECEIVERS}

\section{A. EN 50248}

To test the consumer T-DAB receivers, we first captured the digital I/Q output of a Plisch DAB transmitter. This output contains a modulated digital baseband DAB signal. For band III consumer experiments, DAB mode I was captured; for the L-band, mode IV was captured. See also Fig. 1. After file capture, the appropriate segment was selected. This segment can be converted to a file with or without channel model, or it can be converted into an interference file. The generated files are used in the setup of Fig. 2.

The EN 50248 [4] is an European standard and describes the DAB receiver characteristics. These requirements include minimum performance levels for several parts of the receiver such as power supply, audio and RF performance. In the RF part, the standard defines minimum performance levels for sensitivity, maximum input power, and selectivity (adjacent channel interference and non-adjacent channel interference). The minimum performance level is the level where the BER is equal to $10^{-4}$. So in case of the sensitivity test, the power of the input signal is reduced until the BER reaches this value.

Moreover, the EN 50248 also describes the measurement method for determining these RF performance levels. In this paper we have followed the measurement method of EN 50248. However, all RF performance requirements require Bit Error Rate (BER) measurements and unfortunately, most consumer receivers do not have BER measurement capabilities. Therefore, we cannot use these measuring methods directly in the T-DAB receiver experiments. Also the Pseudo channel BER [5] measure cannot be used in these experiments, because this method requires access to the bitstream before and after error correction. In most DAB consumer receivers these signals are not available.

Instead of measuring the BER, other methods have to be used. The audio quality is affected by the BER and can for that reason be used as a replacement for the BER requirements. Moreover, all receivers can be evaluated using the same measurement procedure as the audio output is available in every receiver. The audio quality can be determined by experienced listeners or by software. We have chosen for the latter method, because it allows automated tests. The received audio signals are compared with the original audio signal using Perceptual Evaluation of Audio Quality (PEAQ) measurement software [6], which is also used in evaluation of audio codecs. The method uses the Objective Difference Grade (ODG) as a measure for the perceptual difference between the recorded receiver audio signal and the original audio signal. The ODG scale ranges from 0 to -4 , see Table I. 
TABLE I

ObJeCtive DifFERENCE GRADE (ODG) SCALE

\begin{tabular}{cl}
\hline ODG & Description of impairments \\
\hline 0 & Imperceptible \\
-1 & Perceptible but not annoying \\
-2 & Slightly annoying \\
-3 & Annoying \\
-4 & Very annoying \\
\hline
\end{tabular}

Fig. 2 depicts the setup for the RF experiments. PC1 and PC2 have an Adlink digital I/O PCI card and can generate in real-time a baseband DAB signal. For Digital-to-Analog (DA) conversion, we used custom-built DA boards; for up-conversion the Agilent $\mathrm{E} 4438 \mathrm{C}$ generator and Rhode \& Schwarz SM300 were used. In both setups, PC 3 is the master computer which controls all other equipment. It also captures the analog audio output of the Device Under Test (DUT) and calculates the ODG metric for each run.

The setup was calibrated by using a Rhode \& Schwarz ESPI [7] to measure the RF output.

\section{B. Test Procedure}

In each experiment, a DAB signal with a center frequency of $225.648 \mathrm{MHz}$ (channel 12B) is used for band III and for the L-band $1464.944 \mathrm{MHz}$ (channel LH). Besides the 'no channel' model, EN 50248 defines two Rayleigh channel profiles. These are a COST 207 Typical Urban channel profile with a velocity of $25 \mathrm{~km} / \mathrm{h}$ and a COST 207 Rural Area channel profile with a velocity of $120 \mathrm{~km} / \mathrm{h}$ [8]. For a single RF signal condition, 15 runs are carried out and for each run, a different realization of the channel model is used. Moreover, in each run the audio is recorded for 25 seconds.

For band III, the coherence time is $73.7 \mathrm{~ms}$ for the urban channel model and $12.5 \mathrm{~ms}$ for the rural channel model. For the L-band these values are $8.9 \mathrm{~ms}$ for the urban model and $4.6 \mathrm{~ms}$ for the rural model. (The coherence time is the time that the channel can be considered as invariant.) This means that for the channel with the largest coherence time, still more than 300 different channels are simulated for each run of 25 seconds and in total more than 5000 .

The audio quality is calculated from the concatenated recorded audio signals, which ensures that the measurement period is long enough to determine an average audio quality. In the figures shown below, one can see that even at good signal conditions, the ODG is unequal to zero. In addition, we have seen that the maximum ODG differs for each receiver. Therefore, we assume that it is caused by the analog amplifier circuit of the audio decoder. In all experiments, the ODG is good until a particular point and then drops very fast. For that reason, we have chosen to use an ODG threshold value of -1 : A single RF condition will pass if the ODG is above this value.

\section{BAND III RESULTS}

In this section, the results of the band III consumer receiver are discussed. First, the results of the antenna factor measurement are described which is followed by the RF experiments of the EN 50248 standard.

\section{A. Antenna Factor}

At ASTRON 5 , Dwingeloo, the Netherlands we measured the antenna factor of consumer whipe antennas for band III in an Anechoic chamber. L-band antennas are always internal antennas and could for this reason not be measured. For the experiment, we used the antennas of three consumer receivers. In the chamber, a known field strength was applied, and the antenna factor could be determined by measuring the received power. For consumer receiver antennas, a typical antenna factor of $25 \mathrm{~dB}$ was found.

\section{B. Sensitivity Experiment}

The sensitivity experiment is used to determine the ability of a receiver to receive weak signals. EN 50248 defines a minimum sensitivity requirement of $-81 \mathrm{~dB}(\mathrm{~mW})$ in a Gaussian channel. In the sensitivity measurement procedure, a DAB signal is applied to the antenna input of the receiver. The power of the signal is reduced until the ODG drops below -1 for a audio sub channel with protection level UEP3. The sensitivity of the receiver is defined as the input power at which the ODG reaches -1 .

Fig. 3 depicts the results of the sensitivity experiment for the rural channel model for the median receiver (solid black line). The audio quality [ODG] is shown along the $y$-axis and the signal power $[\mathrm{dBm}]$ along the $\mathrm{x}$-axis. In addition, three other lines are drawn. The vertical red line is the EN 50248 requirement for this experiment, and the two dashed blue lines are the $25 \%$ and $75 \%$ border lines. The $25 \%$ border means that the best $25 \%$ of the tested receivers have a better performance than this line. The $75 \%$ line is defined in a similar manner. The results for the other sensitivity experiments are listed in Table II. In this table, the 2 nd column list the performance of the median receiver, the 3 rd column the performance of the $25 \%$ worst performing receivers, the 4 th column the performance of the $75 \%$ best performing receivers and the 5th list the EN 50248 requirement.

According Fig. 3, the performance of the median consumer receiver meets the requirements and is at least $10 \mathrm{~dB}$ more sensitive than EN 50248 prescribes.

1) Typical Sensitivity of a T-DAB Consumer Receiver: Another interesting question is whether the minimum sensitivity set by the EN 50248 standard can be achieved by a T-DAB consumer receiver. In this section, the typical achievable sensitivity of a T-DAB receiver is derived.

The sensitivity of a receiver is mainly determined by the combination of three factors:

1) Thermal noise (in semiconductor devices)

2) Noise Figure (NF) of the analog RF front-end

3) The required Signal-to-Noise (SNR) ratio to demodulate a DAB signal

In case of $\mathrm{DAB}$, the signal bandwidth is $1.5 \mathrm{MHz}$ and the noise floor becomes $-112 \mathrm{dBm}$. A typical NF for a DAB front-end in band III and L-band is $6 \mathrm{~dB}$ [9]. In [9], a relation between SNR and BER is given; the minimal SNR for band III is $15 \mathrm{~dB}$ for code rate $1 / 2$ (i.e. protection level UEP3, Mode

\footnotetext{
${ }^{5}$ The radio astronomy organization of the Netherlands.
} 


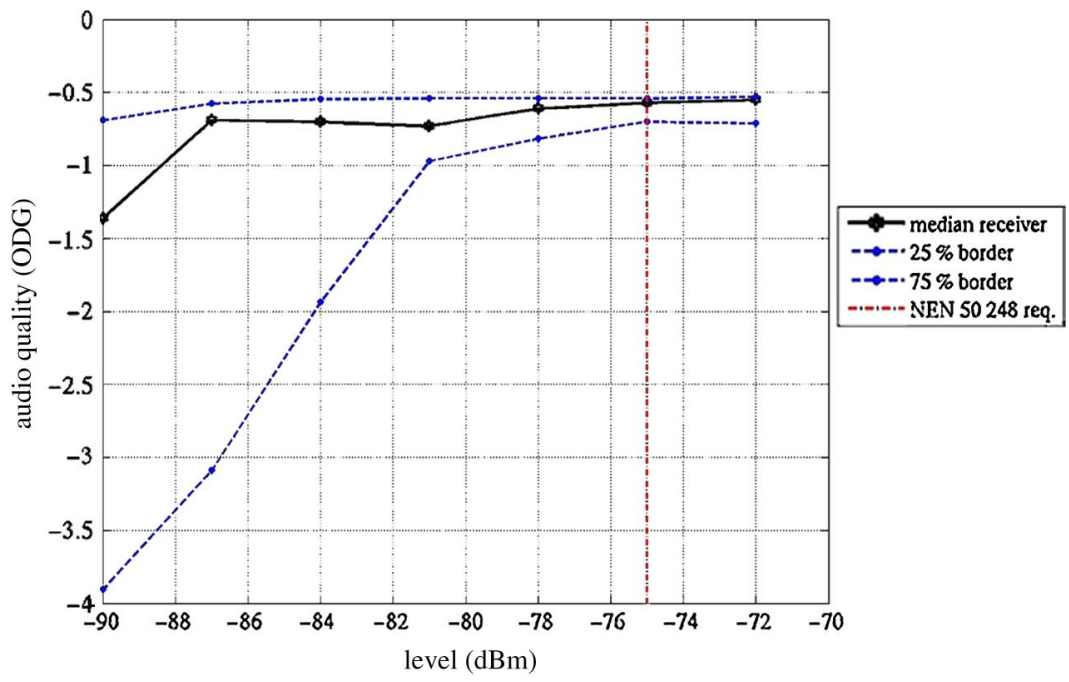

Sensitivity test using COST 207 rural area channel model

Fig. 3. Sensitivity tests of the consumer band III receivers. (a) Sensitivity test using COST 207 rural area channel model.

TABLE II

BAND III RESULTS

\begin{tabular}{|c|c|c|c|c|}
\hline Experiment & $\begin{array}{l}\text { Med. } \\
\text { [dB] }\end{array}$ & $\begin{array}{l}25 \% \\
{[\mathrm{~dB}]} \\
\end{array}$ & $\begin{array}{l}75 \% \\
{[\mathrm{~dB}]} \\
\end{array}$ & $\begin{array}{l}\text { Req. } \\
{[\mathrm{dB}]}\end{array}$ \\
\hline $\begin{array}{l}\text { Sensitivity } \\
\text { no channel }\end{array}$ & $<-90$ & -87 & $<-90$ & $\leq-81$ \\
\hline COST207 urban & $<-90$ & -81 & $<-90$ & $\overline{\leq}-81$ \\
\hline COST rural & -87 & -81 & $<-90$ & $\overline{\leq}-81$ \\
\hline$\overline{\mathrm{ACS}}+1.712 \mathrm{MHz}$ & & & & \\
\hline no channel & -35 & -30 & $>-40$ & $\leq-30$ \\
\hline COST207 urban & -30 & -25 & -35 & $\leq-30$ \\
\hline COST207 rural & -30 & $>-20$ & -35 & $\overline{\leq}-30$ \\
\hline ACS $-1.712 \mathrm{MHz}$ & & & & \\
\hline no channel & -40 & -35 & $<-40$ & $\leq-30$ \\
\hline COST207 urban & -35 & -30 & $<-40$ & $\leq-30$ \\
\hline COST207 rural & -35 & -30 & -40 & $\leq-30$ \\
\hline$\overline{\mathrm{NACS}}+3.424 \mathrm{MHz}$ & & & & \\
\hline no channel & -40 & $>-30$ & -45 & $\leq-40$ \\
\hline COST207 urban & -30 & $>-30$ & -40 & $\overline{\leq}-40$ \\
\hline COST207 rural & -35 & $>-30$ & -40 & $\leq-40$ \\
\hline NACS $-3.424 \mathrm{MHz}$ & & & & -40 \\
\hline no channel & $\begin{array}{c}<-45 \\
-40\end{array}$ & $\begin{array}{c}-35 \\
\geq-30\end{array}$ & $\begin{array}{l}-45 \\
-40\end{array}$ & $\begin{array}{l}\leq-40 \\
<-40\end{array}$ \\
\hline $\begin{array}{l}\text { COST207 urban } \\
\text { COST207 rural }\end{array}$ & $\begin{array}{l}-40 \\
-40\end{array}$ & $\begin{array}{c}>-30 \\
-30\end{array}$ & $\begin{array}{l}-40 \\
-40\end{array}$ & $\begin{array}{l}\leq-40 \\
\leq-40\end{array}$ \\
\hline $\begin{array}{l}\text { In-band int. } \\
\text { no channel }\end{array}$ & 10 & 10 & 10 & - \\
\hline COST207 urban & 15 & 20 & 15 & - \\
\hline COST207 rural & 15 & 20 & 15 & - \\
\hline
\end{tabular}

I) ${ }^{6}$, for the L-band it is $17 \mathrm{~dB}$ for Mode II. (The minimum SNR for mode IV in the L-band is not available in [9], so we assume that for mode IV the same SNR is required.)

Both values are derived for a worst case scenario of a rural area channel model with a speed of $130 \mathrm{~km} / \mathrm{h}$.

The typical sensitivity that a T-DAB receiver can have in band III is the sum of thermal noise, noise figure and required SNR: $-91 \mathrm{dBm}$. The best $25 \%$ of the tested receivers have a similar sensitivity (Fig. 3). For L-band the minimal sensitivity is: $-89 \mathrm{dBm}$. In [10] similar sensitivity values are reported for a DAB chip. Other literature like [3], [11] report better sensitivity

${ }^{6}$ We used protection level UEP3 in our receiver experiments. See also [4]. values, but it is not clear whether this is for a fading channel model.

\section{Adjacent Channel Interference Experiment}

The Adjacent Channel Selectivity (ACS) experiment determines the ability of a receiver to receive a signal when a strong unwanted signal occurs in the frequency band next to the frequency band of the wanted signal. EN 50248 defines a minimum ACS requirement of $\leq-30 \mathrm{~dB}$ when the wanted signal is $-70 \mathrm{dBm}$. The ACS is defined as:

$$
A C S=P_{\text {wanted }}-P_{\text {unwanted }},
$$

where $P_{\text {unwanted }}$ is the signal power of the interfering unwanted signal at which the BER reaches $10^{-4}$.

In total six experiments were performed: an interfering unwanted signal in a channel below (offset $-1.712 \mathrm{MHz}$, channel $12 \mathrm{~A}$ ) as well as in a channel above (offset $+1.712 \mathrm{MHz}$, channel 12C) the channel of the wanted signal. In addition, this was tested for three channel models.

Fig. 4 depicts only the results for the rural area channel model with the $y$-axis showing the audio quality [ODG] and the $\mathrm{x}$-axis showing the power difference between both signals, the Signal-to-Interference Ratio (SIR) [dB]. For other results, see Table II. The median consumer receiver meets the EN 50248 standard for this experiment. The performance of a strong interferer at a higher frequency than the wanted channel is slightly worse than that of a strong interferer at a lower frequency than the wanted channel. In addition, the $25 \%$ worst performing consumer receivers do not meet the requirements for a strong interferer above the wanted channel.

\section{Non-Adjacent Channel Interference Experiment}

EN 50248 also defines a rejection of unwanted signals or far-off selectivity requirement. In the receiver experiment this was replaced by a Non-Adjacent Channel Selectivity (NACS) 


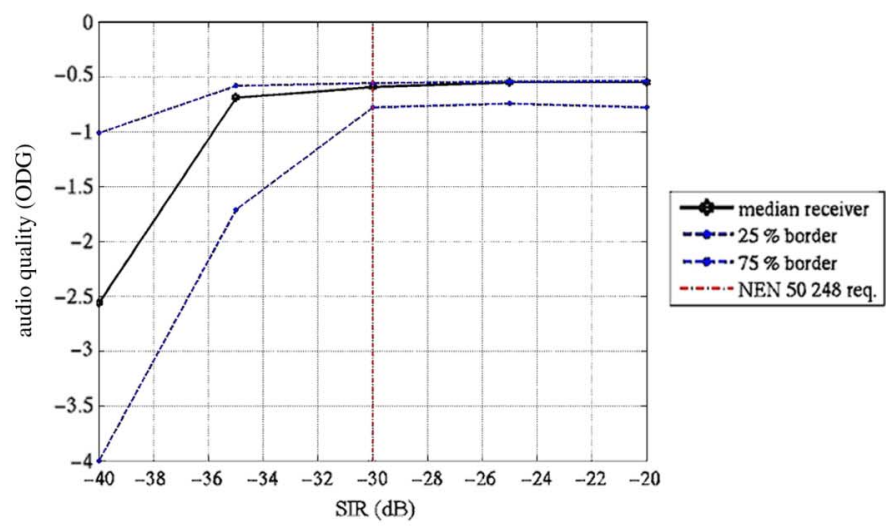

(a)

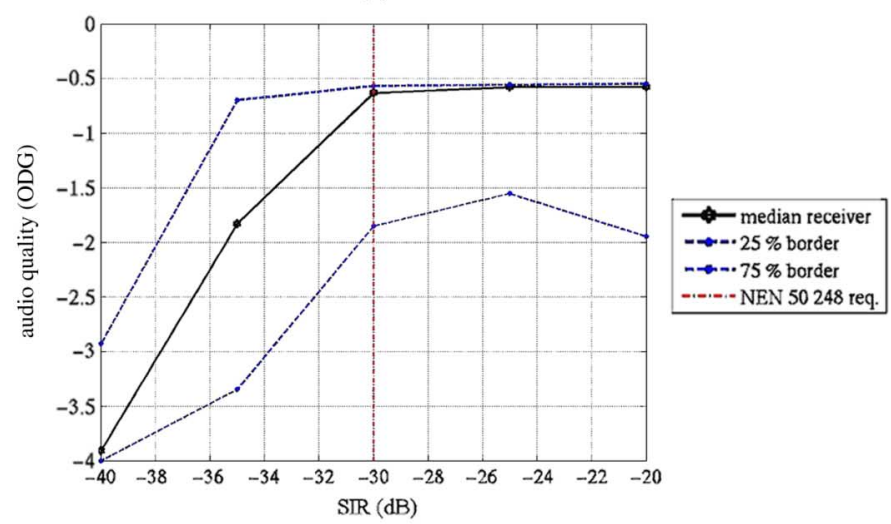

(b)

Fig. 4. Adjacent channel interference tests of consumer band III receivers. (a) Strong interferer at channel 12A using COST 207 rural area channel model; (b) strong interferer at channel 12C using COST 207 rural area channel model.

experiment. The NACS experiment is similar to the ACS experiment. The only differences are that the interfering unwanted signal is in the first non-adjacent channel and that the power levels of the interfering signals are higher. This means that the corresponding NACS requirements are higher as well.

In total six experiments were conducted: a strong non-adjacent interfering unwanted signal in a channel below (offset $-3.424 \mathrm{MHz}$, channel 11D) as well as in a channel above (offset $+3.424 \mathrm{MHz}$, channel 12D) that of the wanted signal. In addition, this was tested for three channel models.

Fig. 5 shows only the results for the rural area channel model with the $y$-axis showing the audio quality [ODG] and the $\mathrm{x}$-axis showing the SIR [dB]. For other results, see Table II. For a strong non-adjacent interferer below the wanted channel, the median receiver just meets the requirements. For a strong interferer at a higher frequency than the wanted channel, the median receiver has up to $10 \mathrm{~dB}$ (for the urban channel model) worse performance than required. Also one can see that the $25 \%$ worst performing consumer receivers have difficulties with all non-adjacent interferer experiments. Only the $25 \%$ best performing receivers meet the non-adjacent requirements set by EN 50248 .

\section{E. In-Band Interference Experiment}

In addition to the experiments based on EN 50248, we also defined an in-band interference experiment. This experiment determines the ability of a receiver to receive a signal with

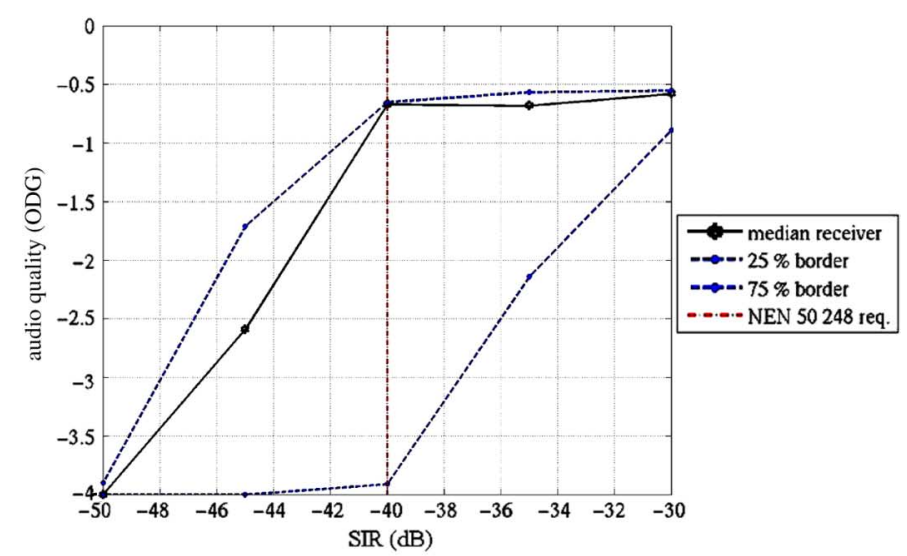

(a)

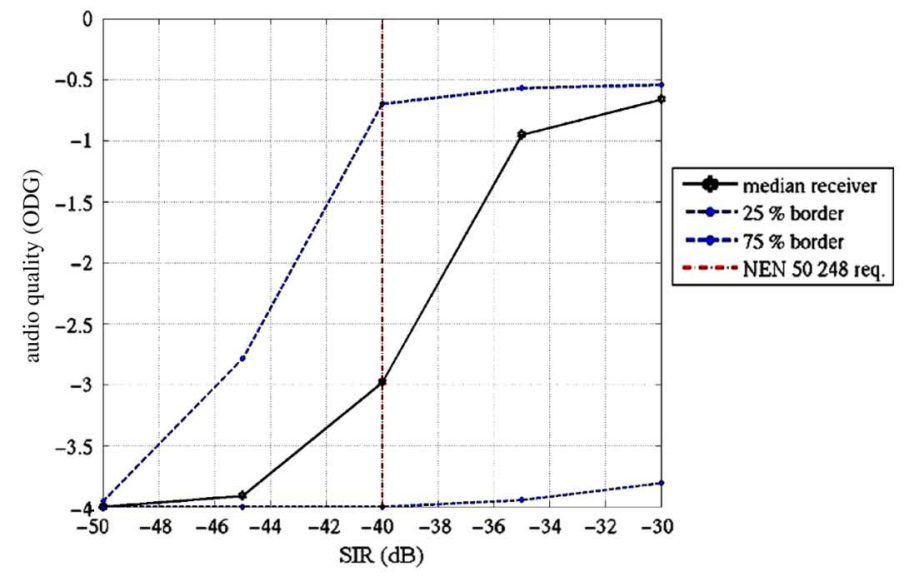

(b)

Fig. 5. Non-adjacent channel interference tests of consumer band III receivers. (a) Strong interferer at channel 11D using COST 207 rural area channel model; (b) strong interferer at channel 12D using COST 207 rural area channel model.

a low signal-to-noise ratio. For simplicity reasons, we added white noise to the wanted channel instead of an in-band interferer. This is valid as an Orthogonal Frequency-Division Multiplexing (OFDM) signal like DAB, resembles white noise. It therefore gives an indication of the ability of a receiver to deal with in-band interference. This value can be used to estimate the frequency re-use distance for T-DAB networks.

Fig. 6 depicts the results of the in-band interference experiment for the rural channel, with the y-axis showing the audio quality $[\mathrm{ODG}]$ and the $\mathrm{x}$-axis showing the SNR [dB]. For other results, see Table II.

The median receiver requires an SNR of about $15 \mathrm{~dB}$. This value is slightly higher than the value found in [12].

\section{L-BAND RESULTS}

The L-band receiver were tested in a similar manner as the band III receivers. The only differences were the channel used (channel LH) and the mode (mode IV).

Both mode II and mode IV are suitable for the L-band, but the latter allows a larger distance between transmitters [13]. For a commercial service, it is likely that mode II will be used instead of mode IV as the latter is only usable for speeds up to 80 $\mathrm{km} / \mathrm{h}$ [14]. However, in this section, the receivers were tested for mode IV as this mode has also been used in the field trial in 


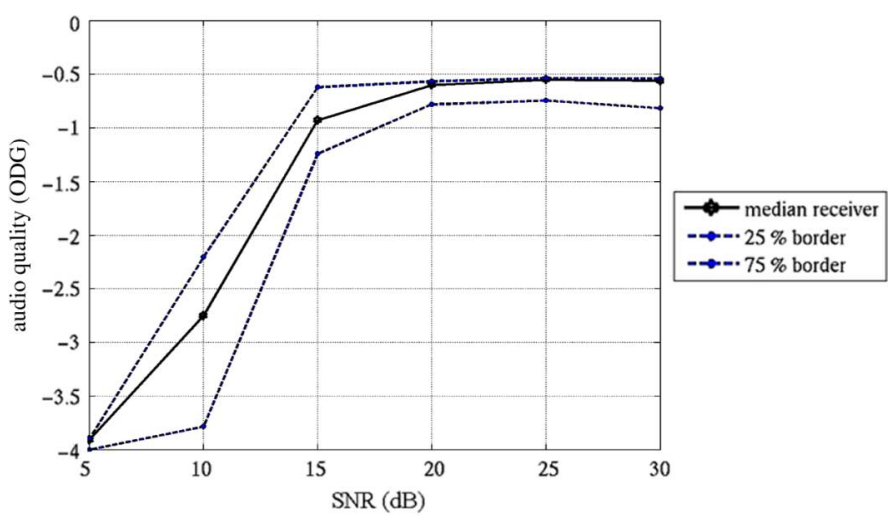

In-band interference using COST 207 rural area channel model

Fig. 6. In-band interference tests of consumer band III receivers (cont). (a) Inband interference using COST 207 rural area channel model.

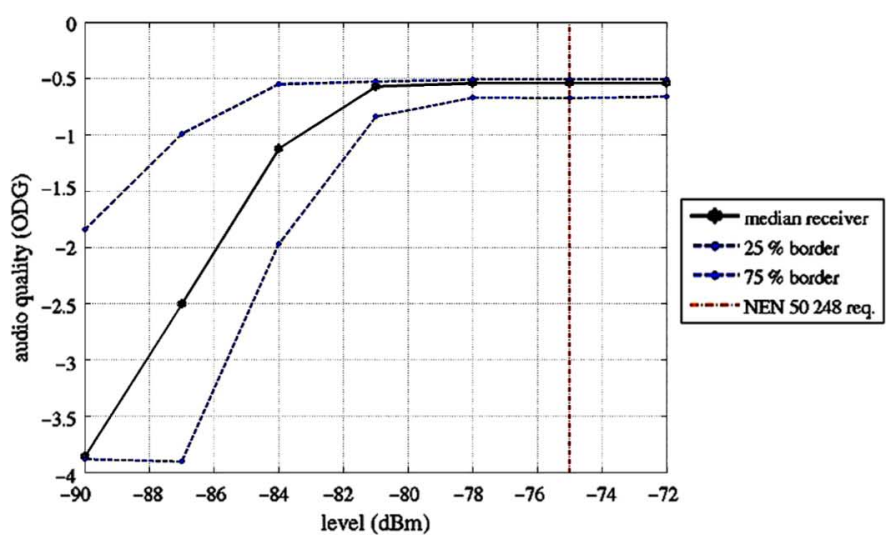

(a)

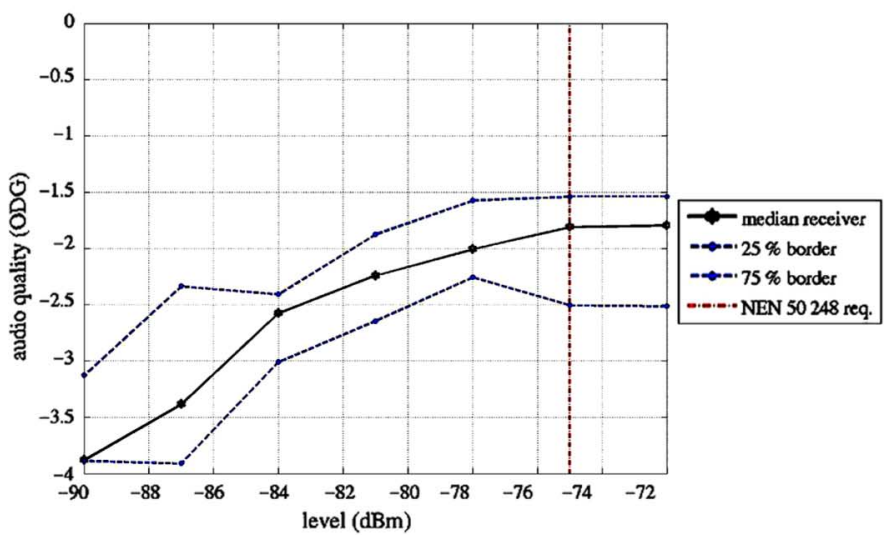

(b)

Fig. 7. Sensitivity tests of consumer L-band receivers. (a) Sensitivity test using COST 207 urban area channel model; (b) sensitivity test using COST 207 ruban area channel model.

Amsterdam [15] $]^{7}$. According to [1], the performance of a consumer receiver depends on the mode used. For that reason, the results shown in this section should be used with care.

\section{A. Sensitivity Experiment}

Fig. 7 depict the results of the sensitivity experiments with different channel models for the median receiver (solid black

\footnotetext{
${ }^{7}$ This research included both receiver experiments and a field trial in Amsterdam.
}

TABLE III

L-BAND RESULTS

\begin{tabular}{l|c|c|c|c}
\hline Experiment & $\begin{array}{c}\text { Med. } \\
{[\mathbf{d B}]}\end{array}$ & $\begin{array}{c}\mathbf{2 5 \%} \\
{[\mathbf{d B}]}\end{array}$ & $\begin{array}{c}\mathbf{7 5 \%} \\
{[\mathbf{d B}]}\end{array}$ & $\begin{array}{c}\text { Req. } \\
{[\mathbf{d B}]}\end{array}$ \\
\hline $\begin{array}{l}\text { Sensitivity } \\
\text { no channel }\end{array}$ & -87 & -84 & $<-90$ & $\leq-81$ \\
$\begin{array}{l}\text { COST207 urban } \\
\text { COST rural }\end{array}$ & -81 & -81 & -87 & $\leq-81$ \\
\hline $\begin{array}{l}\text { In-band int. } \\
\text { no channel }\end{array}$ & 10 & 10 & 10 & - \\
$\begin{array}{l}\text { COST207 urban } \\
\text { COST207 rural }\end{array}$ & $>35$ & 15 & 15 & - \\
\hline
\end{tabular}

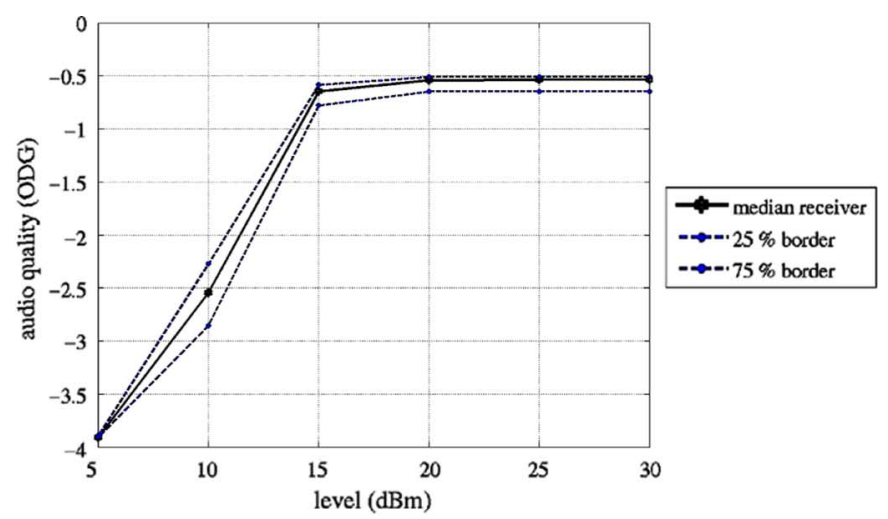

(a)

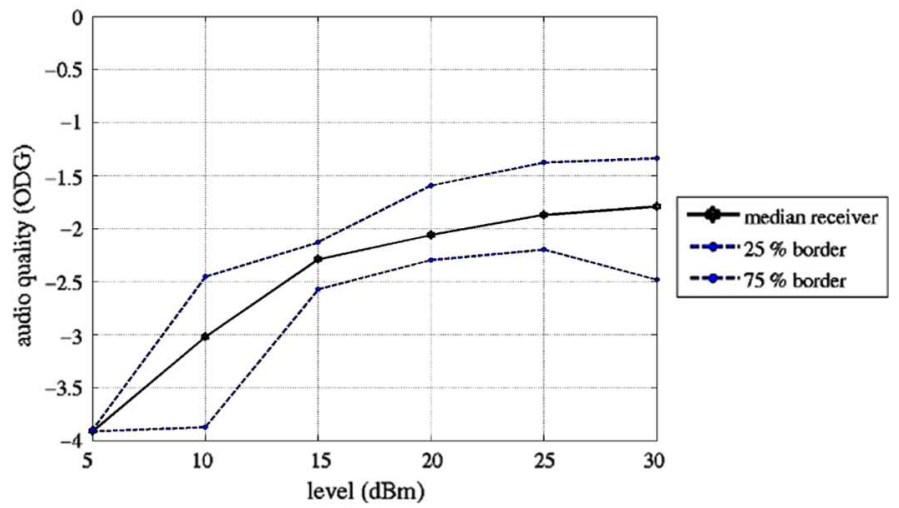

(b)

Fig. 8. (a) In-band interference using COST 207 urban area channel model; (b in-band interference using COST 207 rural area channel model.

line). The $y$-axis shows the audio quality [ODG], and the $\mathrm{x}$-axis shows the signal power $[\mathrm{dBm}]$. See also Table III and Section III-B-1, where the typical sensitivity for a DAB receiver has been derived.

The performance of the median consumer receiver meets the requirements for the no-channel model and the urban area channel. For the rural area channel, the audio quality of the median receiver is too low for good reception.

The coherence time for the COST $207 \mathrm{~L}$-band urban channel is $8.9 \mathrm{~ms}$ and for the rural model $4.6 \mathrm{~ms}$. For differential detection, such as Differential Quadrature Phase Shift Keying (DQPSK), used in DAB, the channel has to be constant over two symbols for proper demodulation. The duration of an OFDM symbol for mode IV is $0.623 \mathrm{~ms}$ [2], so a properly designed $\mathrm{DAB}$ receiver should be capable of decoding the signal for a rural channel model. It is expected that the receivers will have 
a better performance for mode II in the L-band as the OFDM symbol time is smaller (0.312 $\mathrm{ms}$ [2]).

In Canada [1] (2004), the performance of L-band receivers was investigated for mode II. This paper reached similar conclusions. Only one out of six receivers was capable of decoding the signal with the rural area channel model, and three were able to decode the urban area signal. So compared to [1], the performance of the receivers has improved.

In addition, we have carried out a small experiment for a sub channel with protection level UEP3 and mode II. In this experiment, the audio quality of a RF signal level at $-70 \mathrm{dBm}$ has been manually evaluated for a short period. In these experiments no audible audio artifacts were noticed for all L-band receivers. Analysis of the COST 207 channel models support these results.

\section{B. In-Band Interference Experiment}

Fig. 8 depicts the results of the in-band interference experiment with the $y$-axis showing the audio quality [ODG] and the $\mathrm{x}$-axis showing the SNR [dB]. See also Table III.

The median receiver requires a SNR of about $15 \mathrm{~dB}$ for the urban channel mode. This value is less than the SNR value of $18 \mathrm{~dB}$ found in [12].

\section{Conclusions}

In this paper, the RF performance of both band III and L-band T-DAB consumer receivers has been evaluated. The receivers have been tested based on the EN 50248 standard. However, this standard requires BER measurements which is not available in most consumer DAB receivers. For that reason we use the audio quality instead. This audio quality is determined by PEAQ measuring software.

The results show that the median receiver for band III meets the requirements set by EN 50248, except for the non-adjacent interferer experiments. In this experiment, the median consumer receiver performs up to $10 \mathrm{~dB}$ less than required, which of course affects the design of a T-DAB network. In addition, the $\mathrm{RF}$ experiments reveal that there is a large difference in performance between consumer receivers: The $25 \%$ worst performing consumer receivers fail most experiments and the $75 \%$ best performing consumer receivers pass all experiments.

Besides band III, also L-band consumers receiver have been evaluated. The results of the L-band experiments show that the consumer receivers are not capable of decoding a DAB signal with a COST207 rural area channel model in case of T-DAB mode IV. A solution for the designer of a T-DAB network, is to use a different DAB mode, mode II, in the L-band. Preliminary results validate this.

\section{ACKNOWLEDGMENT}

Many people have contributed to this research and the authors thank Henny Kuipers and Geert-Jan Laanstra for construction of the measurement setup. Moreover, the authors thank ASTRON for the antenna factor measurements.

\section{REFERENCES}

[1] A. Carr and B. McLarnon, "DAB-T receiver performance characterisation," in International Broadcasting Conference (IBC), September 2004.

[2] ETSI, "Radio broadcasting systems; digital audio broadcasting (DAB) to mobile, portable and fixed receivers," ETSI EN 300 401, 2001.

[3] H. Su, B. Chi, and Z. Wang, "System design considerations of highly-integrated dab receiver rf front-end," IEEE Trans. Consumer Electronics, October 2005.

[4] "Characteristics of DAB receivers," CENELEC, EN 50248, 1999.

[5] R. Schramm, "Pseudo channel BER-An objective quantity for assessing DAB coverage," EBU Technical Review, vol. 4, 1997.

[6] "Audio quality determination based on perceptual measurement techniques," in Applications of Digital Signal Processing to Audio and AcousticsM. Kahrs and K. Brandenburg, Eds. Boston: Kluwer Academic Publishers, 1998, ch. 1.

[7] [Online]. Available: http://www.rohde-schwarz.com/

[8] M. Pätzold, Mobile Fading Channels. Hoboken, NJ: John Wiley \& Sons, 2002.

[9] "Digital Audio Broadcasting (DAB); Signal Strengths and Receiver Parameters; Targets for Typical Operation," ETSI, ETSI EN 101 758, 2001.

[10] K. Lim, S. Min, M.-W. Hwang, S. Lee, T.-J. Kim, S. Beck, S. Ock, J.-C. Lee, H. Jung, S. Hong, M. Ahn, H. Song, S. Shin, S. Lee, S. Yoo, J. Kim, and S. Han, "A fully integrated low-if image reject receiver for T-DMB and DAB applications," in Proceedings of the IEEE Symposium on VLSI Circuits, 2007.

[11] M. Bolle, D. Clavin, K. Gieske, F. Hofmann, T. Mlasko, M. Ruf, and G. Spreitz, "The receiver engine chip-set for digital audio broadcasting," in Proceedings of the International Symposium on Signals, Systems, and Electronics, 1998.

[12] Y. Jeong, S. Park, and S. Lee, "Evaluation of protection ratios on COFDM for in-band adjacent-channel digital audio broadcasting," in Proceedings of IEEE Region 10 International Conference on Electrical and Electronic Technology, 2001.

[13] W. Hoeg and T. Lauterbach, Eds., Digital Audio Broadcasting: Principles and applications of Digital Radio, 2nd ed. Hoboken, NJ: Wiley, 2003.

[14] B. Breton, R. Voyer, and D. Jacques, "Coverage design aspects of L-band DAB with transmission modes II and IV," in International Broadcasting Conference (IBC), September 1997.

[15] R. Schiphorst, N. Moseley, A. Aarden, H. Heskamp, and C. Slump, "A T-DAB field trial using a low-mast infrastructure," IEEE Trans. Broadcasting, 2008, accepted for publication. 